Revised version submitted to Apj Aug 16, 2016

Preprint typeset using $\mathrm{I}_{\mathrm{A}} \mathrm{EX}$ style emulateapj v. 5/2/11

\title{
MASS DISTRIBUTION AND BAR FORMATION IN GROWING DISK GALAXY MODELS
}

\author{
Joel C. Berrier ${ }^{1}$ and J. A. Sellwood \\ Department of Physics and Astronomy, Rutgers University, 136 Frelinghuysen Road, Piscataway, NJ 08854, USA \\ Revised version submitted to Apj Aug 16, 2016
}

\begin{abstract}
We report idealized simulations that mimic the growth of galaxy disks embedded in responsive halos and bulges. The disks manifested an almost overwhelming tendency to form strong bars that we found very difficult to prevent. We found that fresh bars formed in growing disks after we had destroyed the original, indicating that bar formation also afflicts continued galaxy evolution, and not just the early stages of disk formation. This behavior raises still more insistently the previously unsolved question of how some galaxies avoid bars. Since our simulations included only collisionless star and halo particles, our findings may apply to gas-poor galaxies only; however the conundrum persists for the substantial unbarred fraction of those galaxies. Our original objective was to study how internal dynamics rearranged the distribution of mass in the disk as a generalization of our earlier study with rigid spherical components. With difficulty, we were able to construct some models that were not strongly influenced by bars, and found that halo compression, and angular momentum exchange with the disk did not alter our earlier conclusion that spiral activity is largely responsible for creating smooth density profiles and rotation curves.
\end{abstract}

Subject headings: galaxies: kinematics and dynamics - galaxies: spiral - galaxies: structure

\section{INTRODUCTION}

Understanding the formation of galaxies is one of the grand challenges of current astronomy (e.g. Somerville \& Davé 2015). In parallel with intense observational programs, such as CANDELS Grogin et al. 2011; Koekemoer et al. 2011), many groups are pursuing simulations of cosmic structure formation that attempt to follow the evolution of both dark matter and baryons from $a b$ initio conditions (e.g. Guedes et al. 2011; Munshi et al. 2013; Snyder et al. 2015; Schaller et al. 2015), while others employ semi-analytic techniques (e.g. Lowing et al. 2015; Somerville. Popping \& Trager 2015). However, these endeavors continue to fall short of providing a successful account of the full range of galaxy properties and scaling relations (e.g. Silk \& Mamon 2012; Weinberg et al. 2015). Here we pursue a complementary strategy of using more limited, and less computationally expensive, simulations to attempt to understand pieces of the dynamics of galaxy formation in order to develop insight into the mechanisms that have led to the presentday structure of disk galaxies.

One of the principal unsolved challenges presented by disk galaxies is the frequency of bars. When observed in the near-IR, very roughly one third are strongly barred, a further one third are weakly barred, while the remainder appear not to contain any bar at all (Eskridge et al. 2000; Marinova \& Jogee 2007; Reese et al. 2007; Buta et al. 2015). Several explanations for the presence or absence of a bar have been proposed, though none gives a convincing explanation for these proportions.

- Ostriker \& Peebles (1973), Efstathiou et al. (1982), and Christodoulou et al. (1995) have tried to tie the existence of bars in disks to halo fraction, but this idea collides with both theoretical

\footnotetext{
${ }^{1}$ Currently at Department of Physics, University of Nebraska Kearney, 2401 11th Avenue, Kearney NE 68849
}

arguments and empirical data. Crucially, these stability criteria were derived for rigid halos and are inadequate when halos are composed of responsive particles, as reviewed below. In addition, Toomre (1981), who elucidated the mechanism of the linear bar instability, argued that bar formation can be prevented by inhibiting feedback through the center (see also Binney \& Tremaine 2008; Sellwood 2013). On the observational side, the great majority of spiral galaxies, both with and without bars, have two spiral arms (Davis et al. 2012; Hart et al. 2016), which is an indicator of a heavy disk (Sellwood \& Carlberg 1984; Athanassoula et al. 1987). Also, were barred galaxies to have heavier disk mass fractions, there would be a systematic offset between barred and unbarred galaxies in the Tully-Fisher relation, which is not observed (Mathewson \& Ford 1996; Courteau et al. 2003, see also Bosma 1996).

- Barazza et al. (2009) found an anti-correlation between bulge half-light and bar frequency which is weak evidence in support of Toomre's (1981) suggestion; feedback through the disk center is prevented by an inner Lindblad resonance that should be present when the disk hosts a massive bulge. However, bulge prominence is not a reliable predictor for the existence of a bar, as many earlytype barred galaxies have dense bulges, and at least some late-type unbarred systems have insignificant bulges and gently rising rotation curves. While Toomre's linear-theory prediction has been demonstrated to work in some careful simulations (Sellwood \& Moore 1999; Sellwood \& Evans 2001), bars still form in other cases (Sellwood 1989; Polvachenko et al. 2016), possibly through nonlinear effects. It is also possible that dense centers have been built up subsequently to the formation 
of a bar, perhaps because of inflows driven by the bar in the disk.

- Bars are inhibited in disks having large degrees of random motion (Athanassoula \& Sellwood 1986). But since spiral activity is even more strongly suppressed, random motion cannot be the explanation for unbarred disks that display spiral patterns.

- Pfenniger \& Norman (1990) argued that bars could be destroyed by central mass concentrations. Studies by Norman et al. (1996), Shen \& Sellwood (2004), Athanassoula et al. (2005) and others have shown that the large central masses are required to destroy bars entirely, perhaps $\gtrsim 5 \%$ of the disk mass. Kormendy \& Kennicutt (2004) and Kormendv (2013) argue both that such central masses can be built up by bar-driven gas inflow and that the consequent destruction of the bar creates a pseudo-bulge. However, unbarred galaxies may have neither classical nor pseudo bulges; Table 1 of Kormendy et al. (2010) lists several well-studied cases.

- Cheung et al. (2013) find, from Galaxy Zoo data, a greater bar fraction in galaxies having lower starformation rate and argue that bar frequency is anticorrelated with gas fraction, as was also reported by Masters et al. (2012). Both these papers use visual ( $r$-band) images in which the overall bar fraction is $\lesssim 30 \%$ (Masters et al. 2011); a substantially larger bar fraction is found in NIR images (e.g. Eskridge et al. 2000; Marinova \& Jogee 2007), which suggests that dust and star formation can mask bars in visual images of gas-rich systems (see Block \& Wainscoat 1991, for a remarkable case). Even with this caveat, Masters et al. (2012) gave a barred fraction of $\sim 40 \%$ in galaxies with low $\mathrm{H}$ I mass fractions and $32 \%$ in galaxies with no detectable neutral hydrogen, implying that unbarred, gas-poor galaxies are not uncommon.

- Finally, bars could be tidally triggered (Bvrd et al. 1986; Noguchi 1987; Gerin et al. 1990; Salo 1991; Romano-Díaz et al. 2008; Gajda et al. 2016). Several studies have examined bar fractions as a function of galaxy environments (Elmegreen et al. 1990; Li et al. 2009; Aguerri et al. 2009; Marinova et al. 2012; Lin et al. 2014), although the largest sample (Skibba et al. 2012) confusingly found that the bar fraction was greatest when galaxies were a few diameters apart and decreased at both larger and smaller separations!

We do not attempt to offer a new explanation for the observed bar frequency here. Instead our purpose is to draw renewed attention to the issue and to show that apparently quite reasonable stellar-dynamical models of growing disks are pathologically unstable to barformation. We do not include an explicit dissipative gas component in our simulations, which some argue is crucial, but our conclusions at least remain relevant to gaspoor disks where the problem persists.
As noted above, disks in live halos form bars far more readily than in the equivalent rigid halo Athanassoula 2002). Sellwood (2016) demonstrated that the increased growth rate of the instability resulted from a torque from the halo acting on the disk even at very low amplitude. The instability in his toy model simulations that lacked bulges remained a standing wave with reflection off the center that differed from the mechanism described by Toomre (1981) only in that the bar is excited still more vigorously due to angular momentum loss to the halo.

Saha \& Naab (2013) also reported that bar growth was strongly influenced by the amount and sense of angular momentum in their live halo models. They found that halos rotating in the same sense as the disk caused bars to grow more vigorously and to reach larger size, whereas counter-rotating halos had the opposite effect. A related result was reported by Sellwood (2016), who showed that the strength of the disk-halo coupling depended on the shape of the velocity ellipsoid in non-rotating halos.

These authors, as well as Athanassoula (2008), stressed that the increased tendency for disks to form bars when embedded in responsive halos invalidates the disk stability criteria that were derived for rigid halos (Ostriker \& Peebles 1973; Efstathiou et al. 1982); yet these obsolete stability criteria are still widely invoked.

The vast majority of barred galaxy simulations have begun with unstable axisymmetric disks that quickly form bars. Such simulations have elucidated the instability, as noted above, and have revealed the strong secular growth of the bar (Athanassoula \& Misiriotis 2002; Martinez-Valpuesta, Shlosman \& Heller 2006). But the implication of this body of work for the formation and evolution of real galaxies is less clear, since the initial unstable models are most unlikely to have arisen in nature. We therefore study the evolution of growing disks, in which the bar instability may assert itself as the disk is being assembled.

In Paper I (Berrier \& Sellwood 2015), we showed that non-uniformities in the surface density of a galaxy, and consequent features in the rotation curve, were smoothed out by spiral instabilities. Using highly idealized simulations that mimicked a growing disk in rigid halo and bulge components, we added particles according to rules that were both somewhat realistic, reflecting inside out disk growth (e.g. Matteucci \& Francois 1989; Wang et al. 2011; Bird et al. 2013), and also quite unrealistic. The rules in the latter category were attempts to create galaxy models having unrealistic rotation curves, but the self-consistent dynamics of the disk always frustrated those efforts. In that paper, we deliberately employed rigid mass distributions to represent the halo and bulge in order to show that the radial rearrangement of mass by spiral activity in the disk was alone responsible for the smoothing effect.

In this paper, we report a generalization of this study to include responsive spheroidal components composed of massive particles. We expected the evolution of the rotation curve to differ when the bulge and halo components are made of responsive matter for just two reasons. First, a responsive component compresses as the disk mass rises, due to deepening of the potential well, and second, angular momentum could be transferred from the disk to the spheroidal components, although the effects of this transfer on the radial mass profile are 
not straightforward. For example, Sellwood (2003) and Colín et al. (2006) reported additional contraction of the halo in barred galaxy models; this occured because angular momentum loss from the inner disk, both to the halo and to the outer disk, concentrated the disk mass and further deepened the central potential. This effect overwhelmed any tendency for the halo density to decrease due to the work done on the halo by friction from the bar.

We here report that bars formed in most of the live halo models that matched the stable, rigid bulge/halo models of Paper I. Our models therefore present additional examples that illustrate the excitation of bars by live halos. Even models having moderately massive, dense bulges, which would be predicted to be stable by normal mode analysis (Zang 1976; Toomre 1981; Binney \& Tremaine 2008; Sellwood 2013), formed bars. We suspect that they formed through trapping by strong spirals as Sellwood (1989) had described, but Polvachenko et al. (2016) offer a quite different explanation for the same behavior in their simulations, and the precise mechanism in these cases therefore deserves a separate study.

Our original objective was simply to compare the evolution of the mass distributions and rotation curves in models with live halos with those in Paper I. However, bar formation became a major issue that has made the entire project a much greater challenge. The formation of bars not only confuses the interpretation of spiraldriven evolution, but our prescription, described in 92.2 of adding fresh particles to the disk on near circular orbits ceases to be appropriate when the potential is strongly non-axisymmetric. Although non-axisymmetric forces decay rapidly at radii outside a bar, we report in 3.3 that bars grow in size when fresh particles are added outside the barred part of the disk! For these reasons, we have tried, with limited success, to avoid bars in our models, and those we have selected to report in 84 are some of the few we have run in which the influence of a bar is minor. We study the dynamical consequences of responsive halos and bulges by direct comparisons with models having rigid halos.

\section{LIVE HALOS}

Here we present results from simulations that resemble those in Paper I, but in which the halo and bulge are represented by live populations of particles. We determine the initial equilibria of these components as follows.

\subsection{Model set up}

Our bulge and halo components are created from spherical mass models having known distribution functions (DFs) when in isolation. We then compute the equilibrium DF of the spherical components in the composite disk, halo, and bulge model by recognizing that adiabatic compression leaves the DF unchanged when expressed as a function of the actions ( $c f$. Young 1980). In the case of spherical components, only two actions need be considered: the radial action and the total angular momentum. Details of the iterative solution for a single equilibrium spheroid with an added disk were given in Sellwood \& McGaugh (2005) and its generalization to include multiple spherical components is described here in the Appendix.
The models we present are listed in Table 1, which are selected from the $\sim 100$ simulations we have run in the course of this project. Almost every one of our models having responsive halos formed strong bars. We have opted to present mostly those in which bar formation was sufficiently delayed that the smoothing effect of the spirals could be established.

We employed the Hernquist (1990) sphere for the bulge in some models and for the halo in others. It has the density profile

$$
\rho_{b}(r)=\frac{M q_{b}}{2 \pi b^{3}}\left(\frac{r}{b}\right)^{-1}\left(1+\frac{r}{b}\right)^{-3},
$$

where $r$ is the spherical radius, $M q_{b}$ is the component mass, and $b$ is a length scale. The equilibrium isotropic DF was also given by Hernquist (1990). In other simulations, we used a cored isothermal density profile for the halo, for which we determined the isotropic DF by Eddington inversion (Binney \& Tremaine 2008). The density profile is

$$
\rho_{c}(r)=\frac{M q_{c}}{4 \pi c^{3}} \frac{3+(r / c)^{2}}{\left[1+(r / c)^{2}\right]^{2}},
$$

where $c$ is the core radius and $M q_{c}$ is a mass such that the asymptotic circular speed $V_{\infty}=\left(G M q_{c} / c\right)^{1 / 2}$. As both these halos have infinite extent, we eliminate all particles with enough energy to pass beyond $r_{\max }$, which results in the density decreasing smoothly to zero at that radius.

We employ two types of disk. The usual exponential

$$
\Sigma(R)=\frac{M q_{d}}{2 \pi a_{e}^{2}} \exp ^{-R / a_{e}}
$$

and the Kuzmin-Toomre disk (model 1 of Toomre 1963)

$$
\Sigma(R)=\frac{M q_{d}}{2 \pi a_{k}^{2}}\left[1+\left(\frac{R}{a_{k}}\right)^{2}\right]^{-3 / 2} .
$$

In these expressions, $M q_{d}$ is the disk mass, and $a_{e}$ and $a_{k}$ are length scales. The surface density is tapered smoothly, over half a length scale, to zero at the truncation radius given in Table 1 .

Having determined the equilibrium DF of each spherical component in the composite rigid disk, bulge and halo potential, we used it to select particles as described in the appendix of Debattista \& Sellwood (2000).

We also realize the disk with particles on near circular orbits in the total potential. The initial radial velocity spread is set to create the desired $Q$ value as

$$
\sigma_{R}=Q(R) \sigma_{R, \text { crit }} \quad \text { where } \quad \sigma_{R, \text { crit }}=\frac{3.36 G \Sigma}{\kappa} .
$$

Here $\kappa(R)$ is the local epicyclic frequency. Since the velocity dispersions are a small fraction of the circular speed, $v_{c}$, in our initial sub-maximal disks, the (smaller) azimuthal dispersion can be determined using the epicyclic relation

$$
\sigma_{\phi}=\frac{\kappa}{2 \Omega} \sigma_{R}
$$

where $\Omega(R)=v_{c} / R$. We then use the Jeans equation (Binney \& Tremaine 2008, eq. 4.227) to estimate the 
TABLE 1

SimUlATION INFORMATION - PHYSICAL MODELS

\begin{tabular}{|c|c|c|c|c|c|c|c|c|c|c|c|c|c|c|}
\hline Run & $\begin{array}{l}\text { Disk } \\
\text { type }\end{array}$ & $\begin{array}{l}\text { Disk } \\
\text { edge }\end{array}$ & $\begin{array}{c}\text { Initial } \\
\text { mass }\end{array}$ & $\begin{array}{c}\text { Initial } \\
Q\end{array}$ & $\begin{array}{c}\text { Mass } \\
\text { added } / \tau_{0}\end{array}$ & $\begin{array}{l}\text { Mean } \\
\text { radius }\end{array}$ & $\begin{array}{l}\text { Annulus } \\
\text { width }\end{array}$ & $\begin{array}{l}\text { Bulge } \\
\text { type }\end{array}$ & $\begin{array}{c}\text { Bulge } \\
\text { mass }\end{array}$ & $\begin{array}{l}\text { Bulge } \\
\text { scale }\end{array}$ & $\begin{array}{l}\text { Halo } \\
\text { type }\end{array}$ & $\begin{array}{l}\text { Halo } \\
\text { mass }\end{array}$ & $\begin{array}{l}\text { Halo } \\
\text { scale }\end{array}$ & $\begin{array}{l}\text { Halo } \\
r_{\max }\end{array}$ \\
\hline GR & exp & 4 & 1 & 1.5 & -3.3 & 4 & 4 & none & & & Hern & 36 & 10 & \\
\hline GL & exp & 4 & 1 & 1.5 & -3.3 & 4 & 4 & none & & & Hern & 36 & 10 & 30 \\
\hline $\mathrm{GL}^{\prime}$ & exp & 4 & 1 & 1.5 & none & & & none & & & Hern & 36 & 10 & 30 \\
\hline CR & $\mathrm{KT}$ & 5 & 0.1 & 1.5 & -3.69 & 6 & 2 & Hern & 0.9 & 0.2 & Isot & 14.7 & 30 & \\
\hline CR & & & & & 8 & 2 & & & & & & & & \\
\hline CR & & & & & 10 & 2 & & & & & & & & \\
\hline CR & & & & & 12 & 2 & & & & & & & & \\
\hline CL & $\mathrm{KT}$ & 5 & 0.1 & 1.5 & -3.1 & 6 & 2 & Hern & 0.9 & 0.5 & Isot & 14.7 & 30 & 150 \\
\hline $\mathrm{BL}$ & $\mathrm{KT}$ & 5 & 0.667 & 1.5 & -2.69 & 12 & 4 & Hern & 0.9 & 0.5 & Isot & 14.7 & 30 & 150 \\
\hline $\mathrm{B}^{\prime} \mathrm{L}$ & $\mathrm{KT}^{*}$ & 5 & 0.2 & 1.5 & -2.62 & 7 & 2 & Hern & 0.9 & 0.5 & Isot & 14.7 & 30 & 150 \\
\hline $\mathrm{G}^{\prime} \mathrm{L}$ & $\exp$ & 4 & 1 & 1.5 & -2.3 & 6 & 2 & none & & & Hern & 36 & 10 & 50 \\
\hline $\mathrm{G}^{\prime} \mathrm{R}$ & exp & 4 & 1 & 1.5 & -2.3 & 6 & 2 & none & & & Hern & 36 & 10 & 50 \\
\hline
\end{tabular}

Note. - Column 1: Letter identification for the simulation, followed by either $\mathrm{R}$ for rigid, or L for live halo. Column 2: Type of initial disk; "exp" is exponential (eq. 3) and "KT" is the Kuzmin-Toomre disk (eq. 4) and the asterisk indicates a modification that is described in the text. Column 3: Initial outer radius of the disk in units of $a$. Column 4: Initial disk mass $q_{d}$. Column 5: Initial $Q$. Column 6: Log of fraction of unit mass $M$ added per dynamical time $\tau_{0}$. Column 7: Mean radius of added particles. Column 8: Width of the annulus. Column 9: Type of bulge, if one is present, "Hern" is the Hernquist sphere (eq. 1. Column 10: Mass of the bulge component $q_{b}$. Column 11: Radial scale of the bulge component $b / a$. Column 12: Type of halo, "Hern" is the Hernquist sphere (eq. 1), "Isot" is the cored isothermal sphere (eq. 21). Column 13: Mass of the halo component $q_{b}$ or $q_{c}$. Column 14: Radial scale of the halo component $b / a$ or $c / a$. Column 15: Outer edge of halo, $r_{\max } / a$

TABLE 2

SimULATION INFORMATION - NUMERICAL PARAMETERS

\begin{tabular}{|c|c|c|c|c|c|c|c|c|}
\hline Run & $\begin{array}{l}\text { Cylindrical } \\
\text { polar grid }\end{array}$ & $\delta z$ & $\begin{array}{c}\text { Softening } \\
\text { length }\end{array}$ & $\begin{array}{l}\text { Spherical } \\
\text { grid }\end{array}$ & $\begin{array}{l}\text { Time } \\
\text { step }\end{array}$ & $\begin{array}{l}\text { End } \\
\text { time }\end{array}$ & $\begin{array}{c}\text { Initial } \\
\text { particles }\end{array}$ & $\begin{array}{c}\text { Final } \\
\text { particles }\end{array}$ \\
\hline GR & $106 \times 128 \times 135$ & 0.1 & 0.2 & & $1 / 100$ & 8000 & 6 & 6.7 \\
\hline GL & $106 \times 128 \times 125$ & 0.02 & 0.2 & 301 & $1 / 100$ & 8640 & 6 & 6.7 \\
\hline $\mathrm{GL}^{\prime}$ & $100 \times 128 \times 125$ & 0.02 & 0.2 & 301 & $1 / 100$ & 1500 & 6 & 6 \\
\hline $\mathrm{CR}$ & $128 \times 128 \times 135$ & 0.1 & 0.2 & & $1 / 100$ & 50000 & 5 & 6.71 \\
\hline $\mathrm{CL}$ & $224 \times 256 \times 125$ & 0.1 & 0.2 & 1001 & $1 / 200$ & 2500 & 6 & 7.14 \\
\hline $\mathrm{BL}$ & $224 \times 256 \times 125$ & 0.1 & 0.2 & 1001 & $1 / 640$ & 2250 & 6 & 6.83 \\
\hline $\mathrm{B}^{\prime} \mathrm{L}$ & $100 \times 128 \times 125$ & 0.1 & 0.2 & 501 & $1 / 400$ & 1100 & 6 & 7.15 \\
\hline $\mathrm{G}^{\prime} \mathrm{L}$ & $100 \times 128 \times 125$ & 0.02 & 0.2 & 301 & $1 / 100$ & 2000 & 6 & 6.8 \\
\hline $\mathrm{G}^{\prime} \mathrm{R}$ & $100 \times 128 \times 125$ & 0.02 & 0.2 & 301 & $1 / 100$ & 2000 & 6 & 6.8 \\
\hline
\end{tabular}

Note. - Column 1: Identification, as column 1 of Table 11 Column 2: number of rings, spokes and planes in the cylindrical polar grid. Column 3: Vertical distance between grid planes. Column 4: Gravity softening length, Newtonian forces apply at distances greater than twice this length. Column 5: The number of shells in the spherical grid. Column 6: The basic time step in units of $\tau_{0}$. Column 7: The duration of the simulation. Column 8: Log of initial number of disk particles. Column 9: Log of final number of disk particles.

mean orbital speed $\overline{v_{\phi}}$

$$
{\overline{v_{\phi}}}^{2}=v_{c}^{2}-\sigma_{\phi}^{2}+\sigma_{R}^{2}\left[1+\frac{d \ln \left(\Sigma \sigma_{R}^{2}\right)}{d \ln R}\right] .
$$

Disk particles are spread vertically with a Gaussian profile and the $z$ velocities are determined by integrating the vertical 1D Jeans equation (Binney \& Tremaine 2008, eq. $4222 \mathrm{~b}$ ) for a slab

$$
\sigma_{z}^{2}(R, z)=\frac{1}{\rho(R, z)} \int_{z}^{\infty} \rho\left(R, z^{\prime}\right) \frac{\partial \Phi}{\partial z^{\prime}} d z^{\prime},
$$

which neglects any radial gradients. The vertical gradient of the total potential $\Phi$ is determined from the particles, as well as any additional mass components. Because the initial disks have quite low mass, this standard procedure results in disks that are excellent equilibria, as are the spherical components.

We adopt $M$ as our mass unit, and the disk scale $a$ as the unit of length. Thus our unit of velocity is $V_{0}=$ $(G M / a)^{1 / 2}$ and time unit, or dynamical time, is $\tau_{0}=$ $a / V_{0}=\left(a^{3} / G M\right)^{1 / 2}$. Henceforth, we use units such that $G=M=a=1$. A suitable scaling to physical units for most of our models is to choose $a=0.5 \mathrm{kpc}$ and $\tau_{0}=1.5 \mathrm{Myr}$, which yields $M \simeq 1.2 \times 10^{10} \mathrm{M}_{\odot}$ and $V_{0} \simeq$ $326 \mathrm{~km} / \mathrm{s}$, but other scalings would be more appropriate for some of our models.

\subsection{Disk growth}

As in Paper I and in Sellwood \& Moore (1999), we added particles to the disk mid-plane, giving each zero radial and vertical speeds, with the azimuthal speed $v_{a}=\left(-R a_{R}\right)^{1 / 2}$, where $a_{R}$ is the radial component of the potential gradient at that point and time. This 
procedure loosely mimics the growth of stellar disks, since stars form from gas that has settled into approximate centrifugal balance. The technique was first devised by Sellwood \& Carlberg (1984), and has been adopted recently in other work (Aumer \& Schönrich 2015; Aumer et al. 2016).

This rule gives reasonable initial velocities to the particles only while the potential remains approximately axisymmetric. Since our science goal was to study the consequences of spiral activity, we stop most of our simulations once a dominating bar develops, and our simple rule remains adequate until this moment. Exceptions are presented in 3 .

Not only is our rule computationally more efficient than modeling the hydrodynamics of cooling halo gas, star formation and feedback, but it both gives us far more direct control over the radial location of the added mass and the dynamical consequences are not complicated by "gastrophysics". We exploited that freedom to the full in Paper I, where we placed particles on circular orbits over some adopted narrow radial range.

In all the models reported here, the new particles were added at a constant rate in a narrow annulus with the radial range given in Table 1 . Our objective is not to match any particular expectation of the angular momentum distribution of the material that makes up the disk, but to confirm for live halos what we already showed in Paper I for rigid halos, that the profiles of the evolved disks are insensitive to the distribution of angular momentum added to the disk.

\subsection{Numerical method}

We use the hybrid $N$-body code described by Sellwood (2003, Appendix B), in which the gravitational field is computed with the aid of two grids: a 3D cylindrical polar grid for the disk component, and a spherical grid for the bulge/halo components with a surface harmonic expansion on each grid shell up to order $l_{\max }=4$. A full description of our numerical methods is given in the on-line manual (Sellwood 2014).

We measure the amplitude of non-axisymmetric features by summing

$$
A_{m}(t)=\left|\sum_{j} \mu_{j} e^{i m \phi_{j}}\right|,
$$

for disk particles only, where $\mu_{j}$ is the mass and $\phi_{j}(t)$ the cylindrical polar angle of the $j$-th particle at time $t$. We define the bar amplitude as $A_{2} / A_{0}$. We estimate the length of a bar in a simulation as described in the Appendix of Sellwood \& Debattista (2006). Briefly, it is the average of two separate estimates: (a) the radius at which the $m=2$ amplitude decreases to half its peak value and (b) the radius at which the phase of the same sectoral harmonic differs by more than $20^{\circ}$ from the mass-weighted average near the peak.

We employed one million particles for the halo, and another million of the bulge, if present. The initial and final numbers of particles in the disk are given in Table 2. which also lists the other numerical parameters used for each run. As usual, we checked that outcomes were little changed by reasonable variations of particle number, time step, and grid resolution. Michael Aumer (private
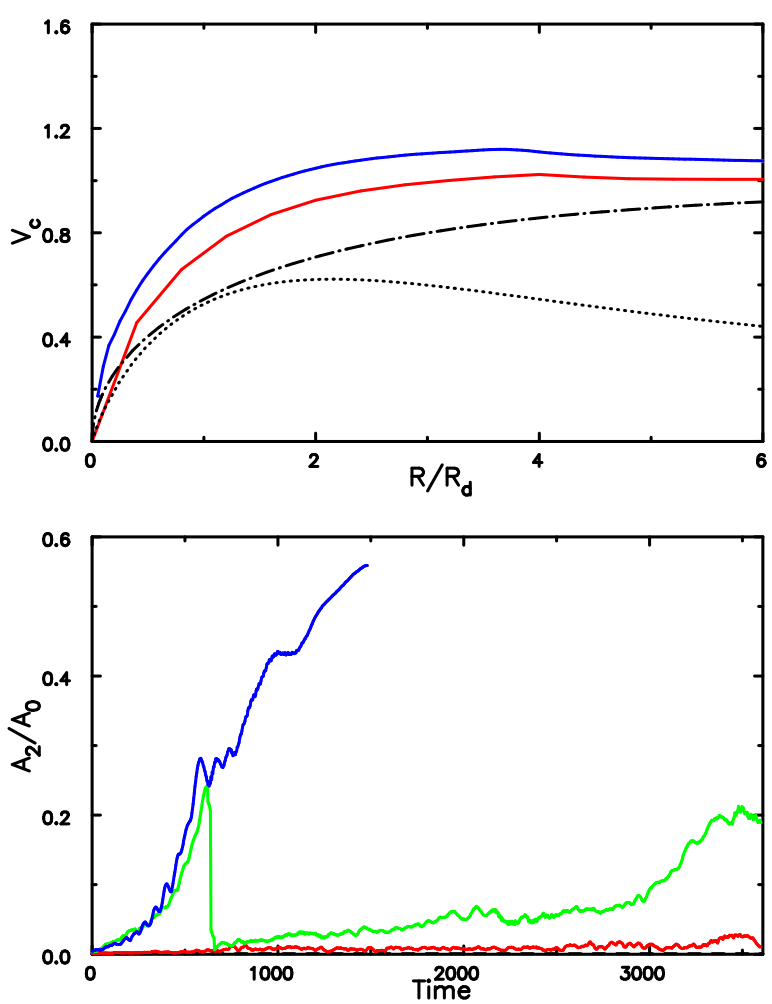

FIG. 1.- Upper panel: the rotation curves of three models without a bulge. The dotted and dot-dash lines show the disk and the rigid halo contributions, while the colored lines show the total circular speed: red for the rigid halo case GR (model G of Paper I), and blue for the live halo GL used here, which employed the same initial halo but which was compressed by the growth of the disk. The lower panel: the time evolution of the bar amplitude. The red line is for model GR, which had a growing disk in a rigid halo, the green line shows the result for GL, a growing the disk in a live halo, with the bar being destroyed by fiat at $t=640$, and the blue line shows $\mathrm{GL}^{\prime}$ the same initial model as GL but with no accretion, showing secular bar growth.

communication) kindly confirmed that responsive halos also lead to higher growth rates for bars when a tree code is used instead.

\section{BAR FORMATION}

\subsection{No bulge}

The upper panel of Figure 1 illustrates the initial rotation curves of three models that consist of a disk and halo only. The red curve is from model GR (the same as model $\mathrm{G}$ of paper I) which was a submaximal exponential disk in a rigid Hernquist halo, as indicated by the dotted and dot-dash lines respectively. The blue curve is for two initially identical models GL that started with the same halo as model GR, but which had been compressed by the growth of the initial disk, and was realized by responsive particles.

The lower panel gives the time evolution of the bar amplitude. The red line shows that the $m=2$ amplitude remained very low in model GR, despite the fact that accretion of particles on near-circular orbits caused the disk mass to grow to 2.8 times its initial mass by the last moment shown, and to 4.5 times the initial mass by the time we ended the calculation. Thus this model appeared to be robustly stable.

The behavior in the live halo is illustrated by the other 


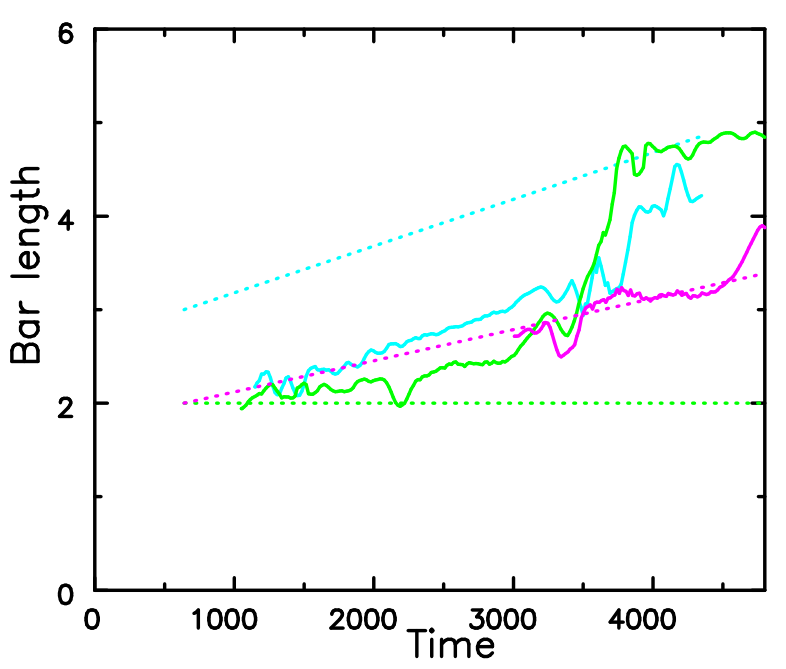

FIG. 2.- The time evolution of the bar semi-major axis in run GL (green), and two similar models. The dotted lines indicate the inner radius of the accretion annulus in the model of the corresponding color. The early evolution to $t=640$ in which a bar formed and was destroyed, as in Fig. 1] is not shown.

two lines. Even though halo compression further reduced the effective mass fraction of the disk, the live halo encouraged rapid bar formation, as found by others. The green line shows model GL, in which we added disk particles by the same rule as in the rigid halo. When we saw that a bar had formed, we destroyed it at $t=640$ by randomizing the azimuths of all the disk particles as we did in Paper I. This intervention therefore created a dynamically hot, featureless disk to which we resumed adding fresh particles. While the hot disk was stable, the effect of the additional cool particles was to make the model become unstable again, and a new bar developed after $t=3000$. The time evolution of the bar length in model GL is shown by the green line in Fig 2, which is described more fully below.

\subsection{Large bars}

One of the major differences between the models used here and those employed by Sellwood (2016) is the much more extensive halo. The blue curve in the lower panel of Fig. 1 illustrates the bar amplitude in model $\mathrm{GL}^{\prime}$ that started out with the same disk and halo as GL, but without the addition of new particles. By the time we stopped the calculation, the bar's semi-axis was $a_{B} \simeq 4 a$, which was equal to the outer radius of the initial disk! This continued secular growth of the bar was caused by ongoing angular momentum exchange with the extensive halo; the loss of angular momentum allowed more disk particles to become trapped into the bar.

Model GL' therefore manifests the same continuous growth of bar size and strength that has been reported previously Athanassoula \& Misiriotis 2002; Martinez-Valpuesta, Shlosman \& Heller 2006). We have verified that a model set up to be as similar as possible to the "massive halo" (MH) case presented by Athanassoula \& Misiriotis (2002) did indeed evolve with our quite different code in the manner they reported. In particular, the bar continued to grow to $t=900$, by which time it was both long (semi axis $\sim 5 a$ ) and very strong $\left(A_{2} / A_{0} \sim 0.5\right)$, as the similar case $\mathrm{GL}^{\prime}$ shown by the blue curve in Fig. 1 .

\subsection{Disk excitation of bars}

While the discussion above has focused on halo excitation of bars, we here report that the cool outer disk also shares the responsibility for large strong bars. In particular, it seemed possible to us that the excitation of a new bar in model GL was caused by too small an inner radius of the accretion annulus, at $R=2$. The added material could couple gravitationally to the inner disk in such a way as to excite a bar. We therefore tried two further experiments in which nothing was changed except that we increased the mean radius of the accretion annulus continuously to larger radii, in the hope that the later added particles would be too far out to couple to the inner disk, thereby avoiding a bar.

However, the models still formed strong bars and, if anything, the bar length seemed to increase with the radius of the accretion annulus, as shown in Figure 2.

The results from model GL are displayed in green in Figure 2] as are those in Figure 1. In this model the inner edge of the accretion annulus was held fixed, and therefore some particles that were added within the bar, which always extended into the accretion annulus, were given inappropriate velocities. In an attempt to avoid this unrealistic evolution, we increased the inner radius of the annulus over time as shown by the dotted lines. In the first such experiment, indicated by the magenta lines, a bar still formed and its semi-major axis seemed to track the inner edge of the annulus, at least for a while. The behavior in an additional experiment that began with a larger accretion annulus that was also increased a little more rapidly is shown by the cyan lines; once again a bar formed whose size rapidly approached the inner edge of the accretion annulus.

It seems that additional cool particles in the outer disk were able to accept angular momentum from the bar in the inner disk, causing it to grow in strength and length. Our strategy of shifting this material to larger radii as the bar grew seemed to enable this happen more readily, having the opposite of the desired effect!

\section{SMOOTHING ROTATION CURVES}

In Paper I, we showed that the final mass and angular momentum distributions in the disk were insensitive to the angular momentum of the accreted material. We gave a full description of the smoothing mechanism in that paper. Briefly, we showed that spiral modes rearranged the angular momentum profile of the disk through horseshoe orbits of stars near the corotation radii of the spirals (Sellwood \& Binney 2002), while the spirals were themselves excited by the same features in the density profile.

Since we employed rigid mass distributions for the spherical mass components of those models, our study clearly demonstrated that the angular momenta of added particles could be diffused by spiral activity so as to create largely smooth disk density profiles and rotation curves lacking strong features. The purpose of this follow-up study was to determine how this result might be affected by replacing the rigid spherical components by responsive matter represented by populations of live particles. 

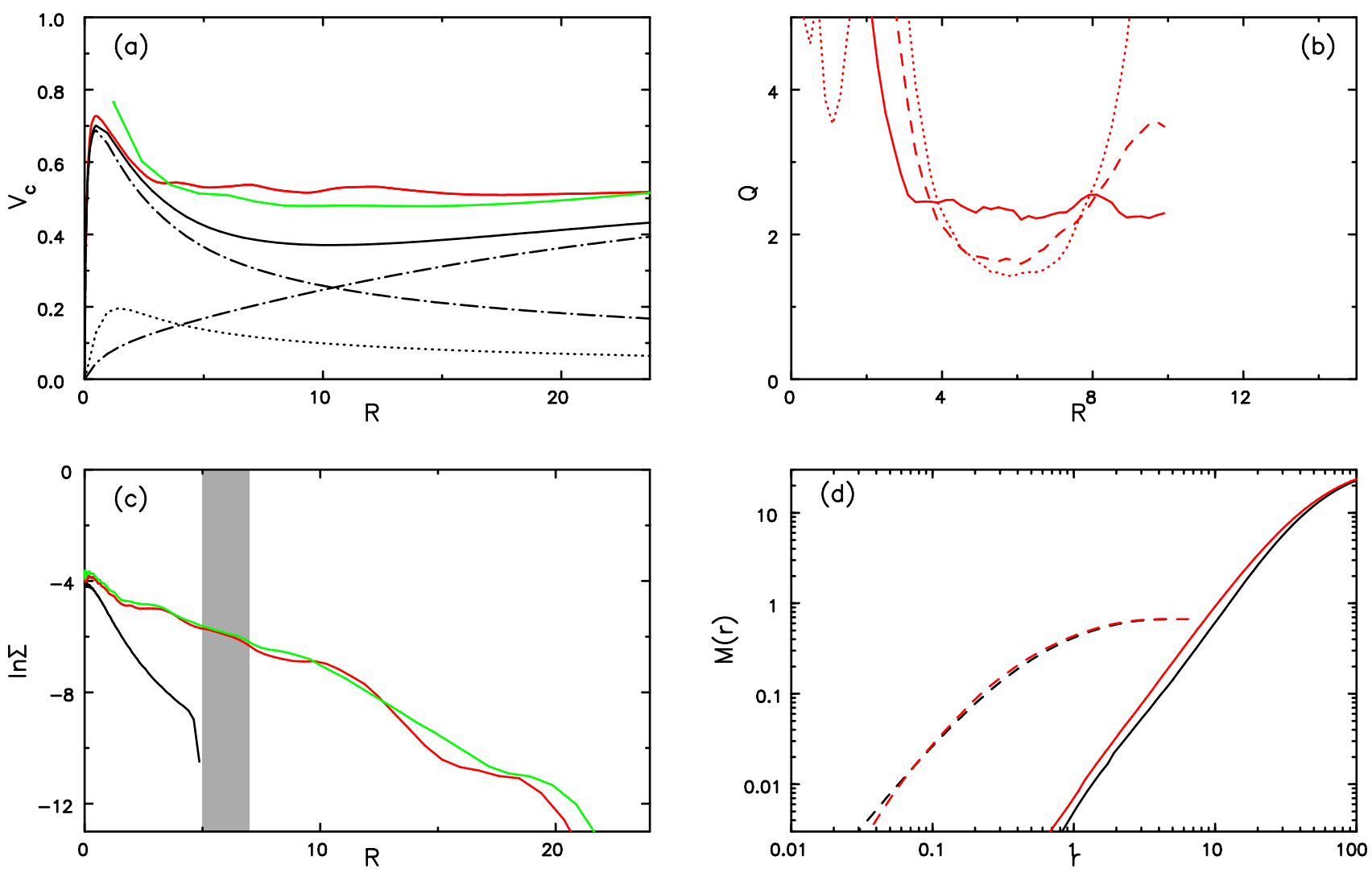

FIG. 3.- (a) The colored curves show the circular speed in run CL (red) at $t=1440$ and in run CR (green) at the same accreted mass. The initial rotation curve is shown by the black lines; the dashed line is the total, with the contributions from the bulge and halo are given by the dot-dash lines, and the disk by the dotted line. (b) The radial $Q$ profiles in model CL at $t=480$ (dotted), $t=960$ (dashed), and $t=1440$ (solid). (c) The initial (black) and final (color) surface density profiles, with shading indicating the radial range of accretion. (d) The mass profiles of the bulge (dashed) and halo (solid) with the initial profile in black and that at $t=1440$ in red.

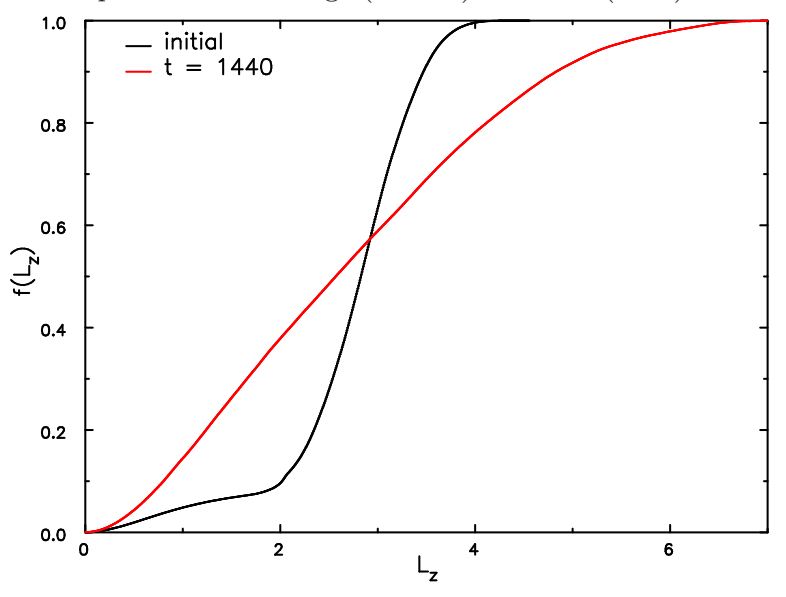

FIG. 4.- Disk mass fraction as a function of angular momentum in run CL. The black curve shows the initial distribution of angular momentum while the red curve indicates the final distribution. Note the smoothness of these distributions, which were measured directly from the large numbers of disk particles (see Table 2).

As previously emphasized, most models from our earlier study with rigid spherical components formed bars when the bulge/halo components were realized with responsive particles. We have opted not to pursue simulations after a strong bar has formed since our science goal is to study the role of spirals alone. We here present only simulations that support a protracted period of spiral ac- tivity, although a bar appeared late in the evolution in most cases, causing us to stop the simulation soon thereafter.

\subsection{Massive bulge}

The red line in Figure 3(a) shows the rotation curve at $t=1440$ from model CL with live bulge and halo components that closely resembled the rigid halo case run $\mathrm{CR}$ (run $\mathrm{C}$ of Paper I); the result from model CR at the same accreted mass is shown by the green line. Both models had a dense Hernquist bulge, a pseudo-isothermal halo, and an initially very low mass exponential disk. We added particles at a constant rate over the radius range $5<R / a<7$, indicated by the shaded region in panel (c), with radii chosen from a uniform probability distribution.

We added mass more rapidly in CL than in CR, did not shift the accretion annulus outwards, and we stopped the simulation at the time shown, when the disk mass had increased 13-fold, because a bar had developed in CL. The bar appeared around $t=1300$ as an oval distortion that extended to $\sim 3 a$ and grew in amplitude (to $A_{2} / A_{0} \sim 0.08$ ) and length (to $\sim 5 a$ ) by $t=1440$.

In this case, the run was able to manifest the smoothing effect of the spirals, as bar formation occurred after much mass had been added. The principal result from Paper I was reinforced: there are no abrupt features in the rotation curve, despite the limited radial extent of accretion. Panel (c) demonstrates that spiral activity spread the added disk material both inwards and out- 

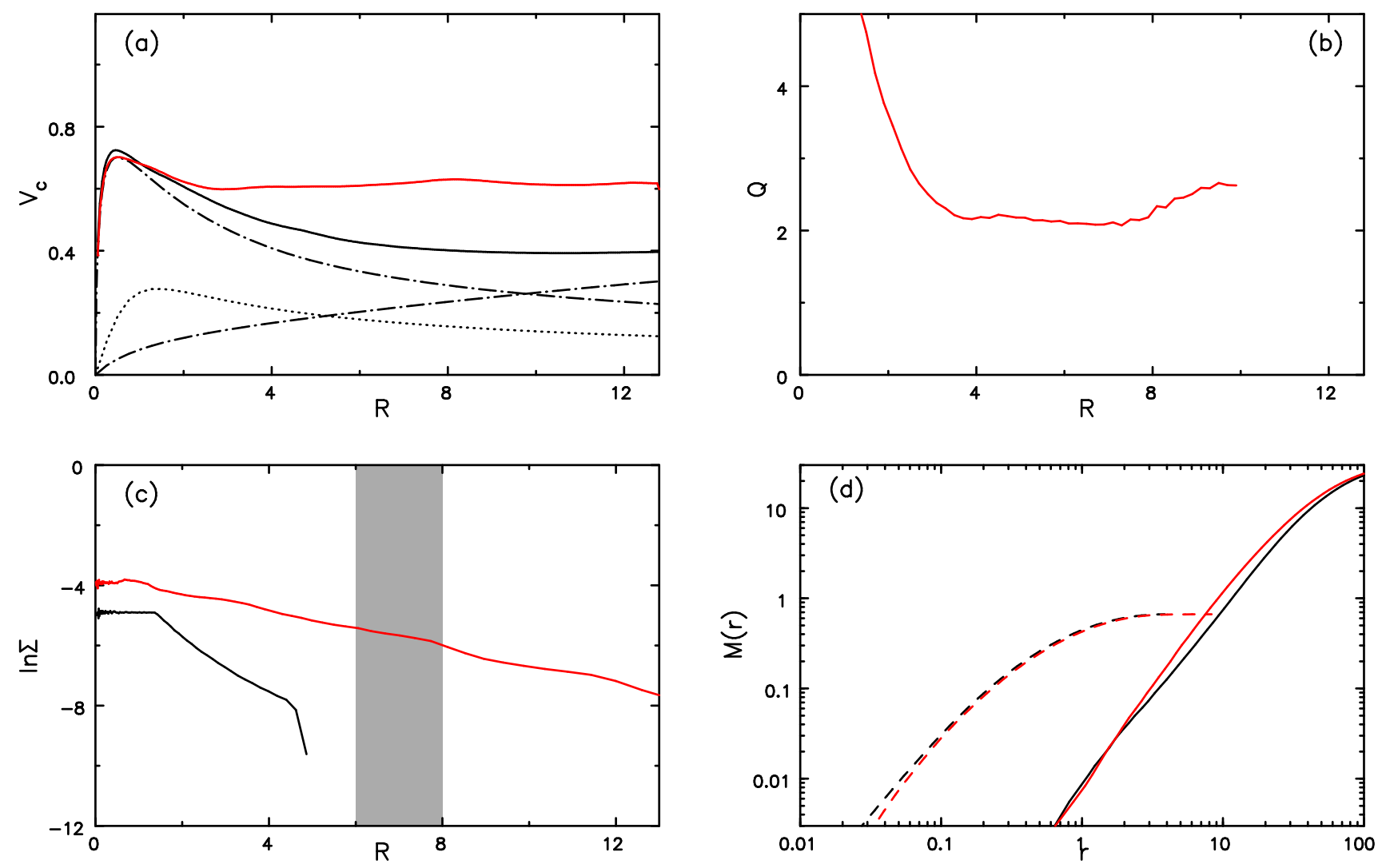

FIG. 5. - As for Fig. 3 but for run $\mathrm{B}^{\prime} \mathrm{L}$ with a live bulge and halo only. The black lines are at $t=0$ and the red lines at $t=1000$.

wards from the annulus in which it was added in a manner very similar to that we found in the rigid halo (green line). Panel (b) shows the $Q$ profile, which was computed from the rms radial velocities and other appropriate disk properties at each radius and time, at three representative times. While the inner disk becomes dynamically hot at an early stage, the region where particles are added stays cool until the bar forms.

Figure 4 confirms that the distribution of angular momentum within the disk is substantially rearranged by the spiral activity. The black line shows the distribution in the initial disk, which extended to $L_{z} \sim 2$ plus the distribution of the added particles, which were placed on circular orbits outside the initial disk. The red line indicates that spiral activity has substantially smoothed the distribution by the time shown. As noted in paper I, the near constancy of $d M_{d} / d L_{z}$ is reminiscent of, but not quite consistent with, the arguments presented by Lovelace \& Hohlfeld (1978).

As expected, halo compression yields a slightly higher circular speed in CL than in CR for the same disk mass added. The solid curves in panel (d) of Fig. 3 show the radial mass profiles of the initial (black) and final (red) halo, indicating the extent of halo compression by the added disk. The dashed curves show the same quantities for the bulge, whose profile is little changed except in the innermost $1 \%$. Very little angular momentum is gained by the halo from the disk in run CL, with the torque increasing to $d L_{z} / d t \simeq 10^{-5}$ by the end as mild bar begins to form. The angular momentum gained by the bulge is at least an order of magnitude less.

\subsection{Moderate bulge}

Run B from paper I had a less massive bulge, and we obtained a similar result in a parallel simulation with a live bulge and halo, although we ended the live halo simulation at $t=8000$, whereas we had continued the rigid halo model to $t=18000$. Since the orbit period at $R=5 a$ is $\simeq 63$ of our time units, the duration of even the shorter integration was some 125 disk rotations, which is considerably longer than the age of a Milky Way-like galaxy.

As for model CL above, we therefore ran other simulations with substantially higher accretion rates, in order to reach the same final disk mass within the expected lifetime of a galaxy. However, this approach was unsuccessful for this lower bulge mass, because the models formed strong bars well before the desired final mass was reached.

One such example was model BL. As in Paper I, we destroyed the initial bar at $t=750$, after which we began to add particles far outside the original disk. However, a new bar formed around $t=1400$ that extended as far as the accretion annulus and grew in amplitude until we stopped the run at $t=2250$ when the disk mass had increased just 5-fold.

Since this behavior prevented us from observing the smoothing effect of spiral activity, we had to develop a strategy that would inhibit the formation of bars. After much experimentation, we found the most effective way to delay the formation of a bar was to reduce substantially the inner disk surface density in the region of the bulge. The evolution run, $\mathrm{B}^{\prime} \mathrm{L}$, is shown in Figure 5. The 

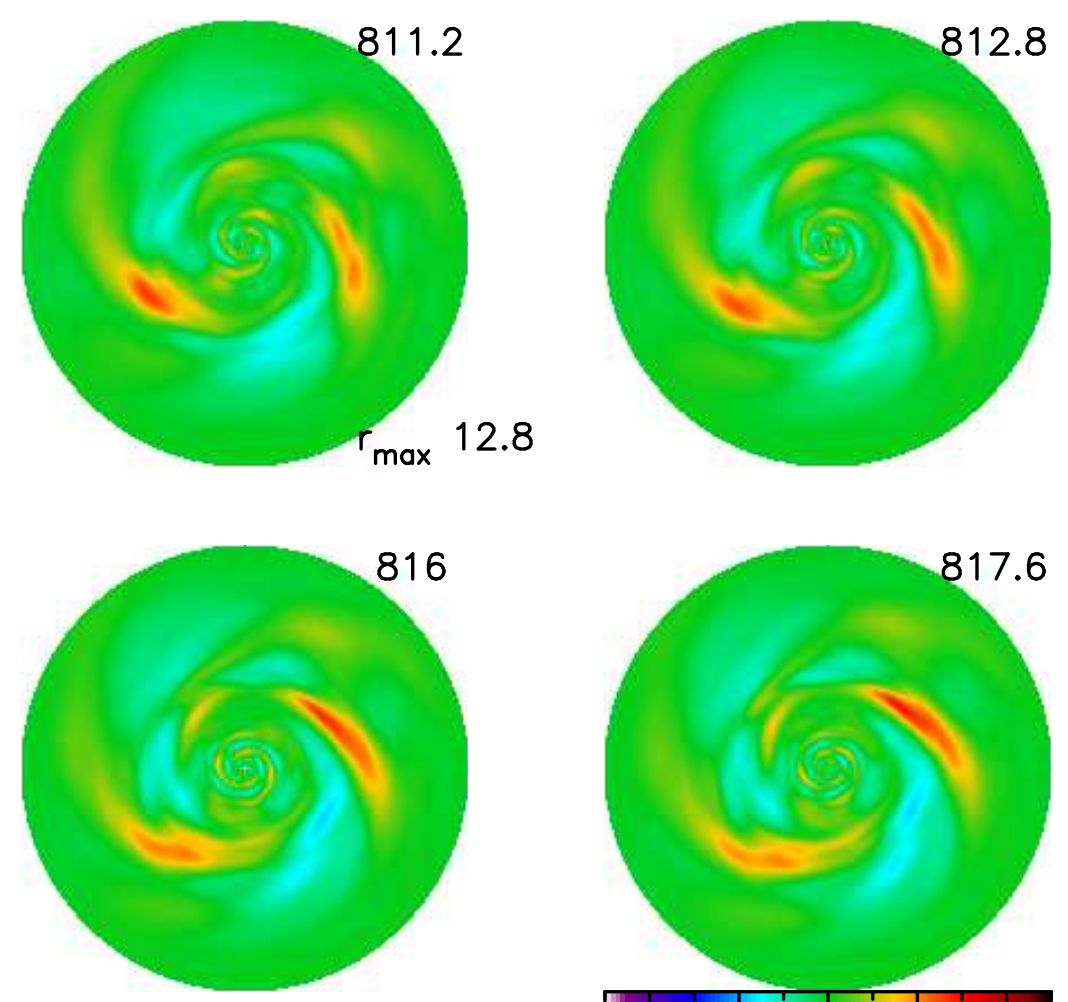
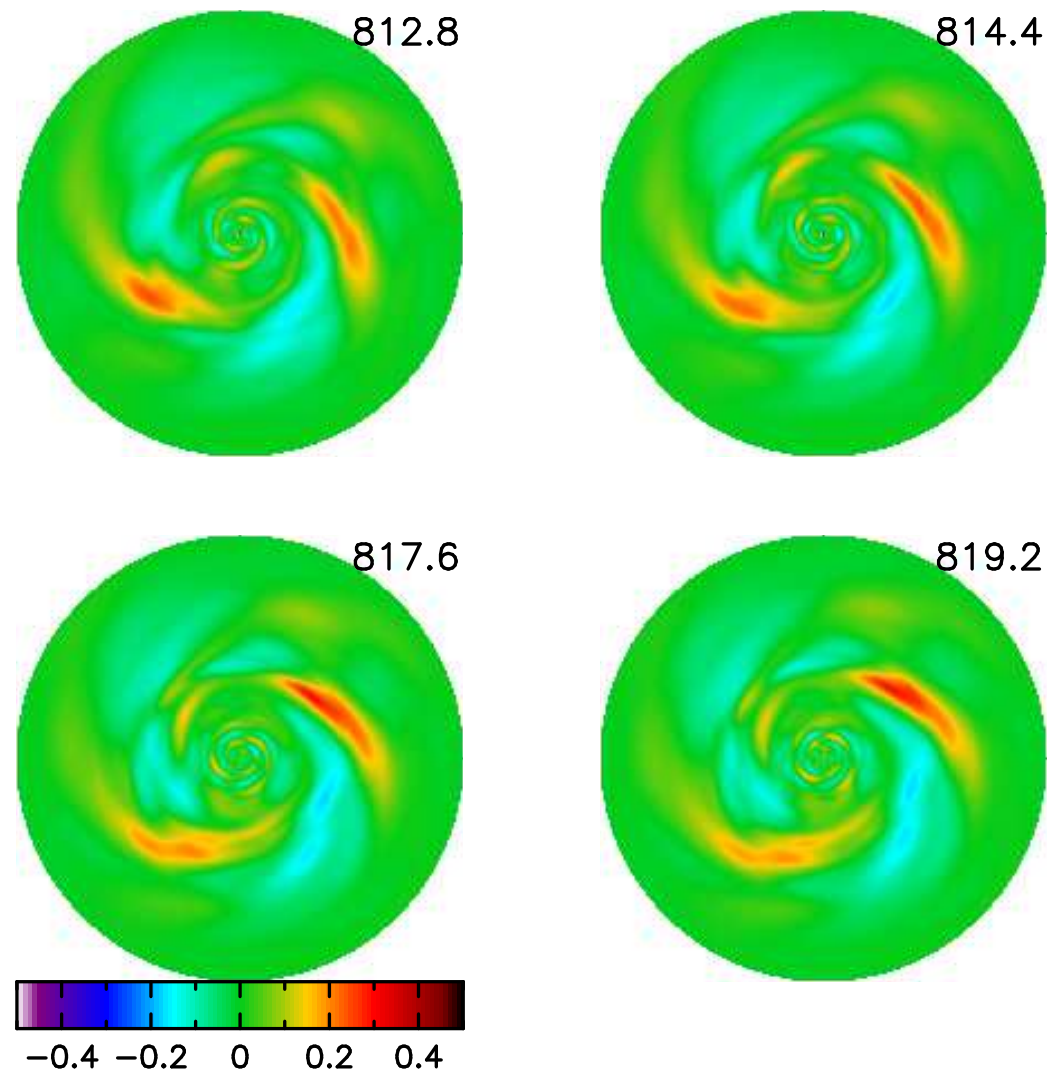

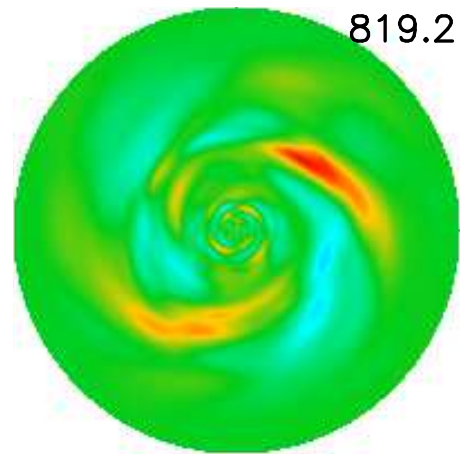

FIG. 6.- A short time sequence late in the evolution of run $\mathrm{B}^{\prime} \mathrm{L}$ showing departures from the mean density at each radius. Note the tightly wrapped spiral in the disk center where the surface density was low.

initial KT disk profile is given by the black line in panel (c), with the surface density held constant when $R \lesssim 1.2$. We added particles at a constant rate from $t=0$ in the annulus $6<R<8$ such that the disk mass increased 13 -fold by $t=1000$.

As expected, lowering the surface density had the effect of decreasing the spatial scale of self-gravitating density waves. Figure 6 illustrates a tightly wrapped $m=2$ spiral in the inner disk over a short interval late in the evolution of run $\mathrm{B}^{\prime} \mathrm{L}$. The radial group velocity of spiral waves (Toomre 1969; Binney \& Tremaine 2008; Sellwood 2013) is $v_{g}=\partial \omega / \partial k$, where $\omega=m \Omega_{p}$ is the pattern speed and $k$ the radial wavenumber. Binney \& Tremaine (2008, their eq. (6.76), which is missing a $\operatorname{sgn}(k)$ factor) give the messy expression for the group velocity derived from the WKB dispersion relation for stellar disks, the leading factor of which is inversely proportional to $k$. In this case therefore, the tighter winding of spiral waves where the surface density is lowered also likely slows the group velocity. Delayed feedback through the center, if it occurs at all, slows the growth rate of the bar instability, substantially delaying the formation of the bar.

The smoothing effect of the spirals again dominated the changes to the circular speed curve (Figure [5]), as well as the surface density profile (c). The surface density of the inner disk remained low for most of the run, but a large bar developed shortly before the time shown $(t=$ 1000) that was associated with a significant increase in the inner surface density. The effect of halo compression is shown in $(d)$.

\subsection{No bulge}

As shown by the red line in Fig. 1(b), model GR (G of paper I) did not form a bar even as the disk mass was increased substantially, but bars formed more readily when the same halo was made responsive (model GL) even without the addition of disk mass (model $\mathrm{GL}^{\prime}$ ). We could have suppressed bar formation by increasing the halo mass fraction (Maver \& Wadslev 2004; Sellwood 2016), but mass rearrangements by the small-scale spirals that would develop within such extreme low mass disks would cause only minor changes to the shape of the overall rotation curve, which would not achieve our science goal.

In Paper I, we dealt with bar unstable disks by letting the disk form a bar and then scrambling the azimuths of the disk particles to erase the bar and using this hot initial disk as the starting point for mass accretion. This growing disk in the rigid halo generally avoided forming a new bar, at least for long enough to reveal the smoothing effects of spirals. However, as shown in Fig. 1] a fresh bar formed in a live halo when we equally slowly added cool material to the hot disk made in this manner.

Thus, following Saha \& Naab (2013), we suppressed the supporting response to the bar mode in the disk by making the halo rotate strongly in the sense counter to disk rotation. This strategy is clearly an artifice: in reality, any halo rotation should have nearly the same sense of rotation as the disk, because both the halo and the disk material were subject to the same tidal torques and inflows in the early universe (Efstathiou 1979; Bullock et al. 2001; Bett et al. 2007). The disk 

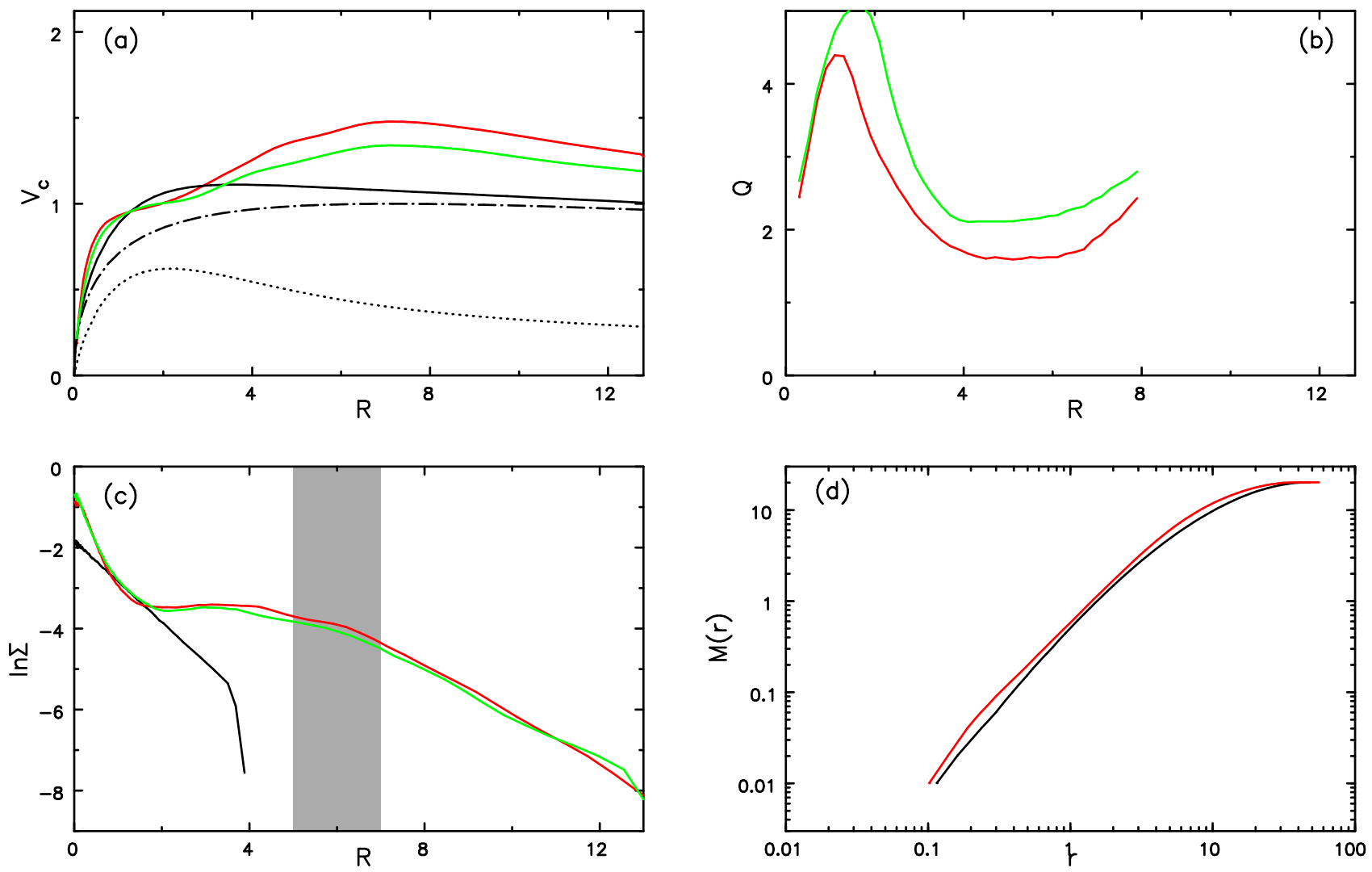

Fig. 7. - As for Fig. 3 but for run $\mathrm{G}^{\prime} \mathrm{L}$ with a halo only and no bulge. The black lines are at $t=0$ and the colored lines at $t=2000$ : red for $\mathrm{G}^{\prime} \mathrm{L}$ with the live halo, green for $\mathrm{G}^{\prime} \mathrm{R}$ in which the halo was frozen.

spins up as it shrinks and settles through dissipation, while rotation in the more extended collisionless halo is also expected to be mild and in the same sense as that of the disk (but see also Danovich et al. 2015).

We added rotation to the halo in the following manner. An isotropic halo has $f(E)$ only, with uniform probability of any allowed value of the total angular momentum $L$ at that $E$. We selected particles with the required distribution of these integrals as described in Debattista \& Sellwood (2000), and then chose a radius at random from a uniformly distributed fraction of the radial period. These choices determine $v_{\perp}$ and $v_{r}$ for the particle, where $v_{\perp}=L / r$ and $v_{r}^{2}=2[E-\Phi(r)]-L^{2} / r^{2}$ are the components of velocity perpendicular and parallel to the radius vector. For an isotropic DF, the direction of $v_{\perp}$ is uniformly distributed in $-\pi \leq \psi \leq \pi$, where $\psi$ is the angle to the $(x, y)$ plane, say. We can add arbitrary amounts of net retrograde rotation by biasing the choice of $\psi$ towards values $|\psi|>\pi / 2$. In our case, we set $\psi=\pi u^{1 / 3}$, where $u$ is uniformly distributed over the range $-1<u<+1$.

Although a rigid halo of this mass is sufficient to inhibit bar formation, even the strong retrograde bias of this live halo, model $\mathrm{G}^{\prime} \mathrm{L}$, did not prevent a cool disk from forming a bar. We therefore destroyed the bar at $t=640$ by randomizing the azimuths of all the disk particles, thereby creating a hot, stable disk. We added particles to this initial, dynamically hot disk over the radial range $5<R / a<7$ at a higher rate than in model GL and no bar formed during the subsequent evolution to $t=2000$, when the disk mass was 6.2 times greater than at the outset. $\mathrm{G}^{\prime} \mathrm{L}$ was our only model with a live halo that had no tendency to form a bar; comparison models with isotropic halos, e.g. GL in Fig. 1] always formed fresh bars during the accretion phase.

Figure 7 shows the results at $t=2000$; the red lines show the behavior of the model with a live halo, $\mathrm{G}^{\prime} \mathrm{L}$, and the green lines illustrate the evolution of run $\mathrm{G}^{\prime} \mathrm{R}$ in which the live halo was frozen at $t=640$ after the initial formation and destruction of the bar. Once again, spiral activity has rearranged the disk radial density profile in almost the same manner for the live and rigid halos, as shown in panel (c). The central density spike was created as the bar formed in the part of the evolution before particles were added. The effects of halo compression are evident in panels (a) and (d); the black line in (d) indicates the halo mass profile at the start of the simulation, while the red shows the profile in $\mathrm{G}^{\prime} \mathrm{L}$ after the disk mass was increased. The difference in the red and green lines in (a) is due almost entirely to the compression of the live halo. As in model $\mathrm{C}$, above, less than $2 \%$ of the final disk angular momentum was given up to the halo over the interval $960<t<2000$.

\section{CONCLUSIONS}

The results presented in the previous section have met our original science objective of showing that a responsive halo does not alter the conclusion from Paper I: the disk density profile and rotation curve of the composite system are naturally smoothed by internal dynamical evolution as a disk grows in mass. It is interesting that the end condition seems to be characterized by a uniform 
distribution of disk mass with specific angular momentum (Fig. 4). Comparison between models with rigid and responsive halos reveals that halo compression plays a more minor role than the spiral activity, which we identified in Paper I as the primary cause of smoothing. We also found that a disk supporting spiral activity does exert a torque on the halo but, unless a bar develops, only a tiny fraction of the total disk angular momentum is given up to the halo.

However, these conclusions were reached only after we were able to overcome the very pronounced tendency for a disk in a live halo to form a bar where a similar disk in a rigid halo did not. This increased tendency for bar formation had been reported by others Athanassoula 2002; Saha \& Naab 2013), and was studied in greater depth by Sellwood (2016) who focused on highly idealized models with halos that were not at all extended.

Since we wished to examine the effects of spirals without a dominating bar, we struggled to create initial models that were not strongly unstable when embedded in responsive halos and bulges. Unless the bulge was extremely massive (Fig. 3), our simulations with dense bulges quickly formed bars, despite the prediction by Toomre (1981) that a dense center should prevent feedback and therefore be stabilizing.

We were able to at least delay the formation of a bar in models with moderate bulges by reducing the central density of the disk (Fig. 5). We also adopted the strategy from Paper I of allowing a bar to form and then destroying it, by randomizing the azimuths of the disk particles, thereby creating a dynamically hot disk (Fig. 1). However, both these strategies provided only temporary respite from bar formation as we increased the disk mass by adding fresh, dynamically cool particles. We were able to weaken the coupling of the disk to the halo by making the two components counter-rotate, an unrealistic strategy that appeared to be the only way to prevent the eventual formation of bar.

We also report (3.2), as was also found previously by Athanassoula \& Misiriotis (2002) and Martinez-Valpuesta. Shlosman \& Heller (2006), that bars in unstable models with extended halos become quite unrealistically large and strong (e.g. Erwin 2005). While the extraordinary secular growth of bars man- ifested by such models is interesting, they start from cool, axisymmetric disks that are strongly unstable i.e. from initial conditions that could not arise in nature.

The dominance of bar formation in our models was indeed remarkable. Almost every live-halo model presented here formed a bar eventually, a result that is apparently inconsistent with the existence of unbarred galaxies in nature, as reviewed in the Introduction.

Cheung et al. (2013) and others (e.g. Bournaud et al. 2005) have claimed that bars can be avoided in simlations having large gas fractions. However, Aumer et al. (2016) reported simulations that included both dissipative gas and heavy particles to represent giant molecular clouds, but found that scattering of star particles by the heavy particles, slowed but did not inhibit entirely, the development of bars in their models. Many simulations of galaxy formation in a cosmic context have included rules to mimic baryonic physics to the most realistic extent possible, and some (e.g. Stinson et al. 2013; Aumer, White \& Naab 2014; Snyder et al. 2015) have reported Milky Way-like galaxy models that do not possess bars. The challenge will be to show not only that these simulations can reproduce the observed bar fraction, but also that the disks in such models are realistically massive, dynamically cool, and thin so that they can also support the generally-observed two-armed spiral patterns.

Since we have employed only collisionless particles, and do not include the physics of gas, our findings may perhaps apply to gas-poor galaxies only. However, a large fraction of such galaxies appear to be unbarred (Masters et al. 2012), and the low gas fractions imply that bars would be harder to mask with star formation and dust (Buta \& Block 2001). Note that while large gas fractions are expected in the early stages of galaxy formation, when we destroyed the early bars, fresh bars formed as we modeled the continued gradual growth of galaxy disks in a physically reasonable manner. Thus the problem is also one of late evolution, and not just of the early stages.

We thank the referee for a valuable report that greatly helped us to clarify the paper. This work was supported by NSF grants AST/110897 and AST/12117937.

\section{REFERENCES}

Aguerri, J. A. L., Méndez-Abreu, J. \& Corsini, E. M. 2009, A\&A, 495, 491

Athanassoula, E. 2002, ApJL, 569, L83

Athanassoula, E. 2008, MNRAS, 390, L69

Athanassoula, E., Bosma, A. \& Papaioannou, S. 1987, A\&A, 179, 23

Athanassoula, E., Lambert, J. C. \& Dehnen, W. 2005, MNRAS, $\mathbf{3 6 3}, 496$

Athanassoula, E. \& Misiriotis, A. 2002, MNRAS, 330, 35

Athanassoula, E. \& Sellwood, J. A. 1986, MNRAS, 221, 213

Aumer, M., Binney, J. \& Schönrich, R. 2016, MNRAS, to appear (arXiv:1604.00191)

Aumer, M. \& Schönrich, R. 2015, MNRAS, 454, 3166

Aumer, M., White, S. D. M. \& Naab, T. 2014, MNRAS, 441, 3679

Barazza, F. D., Jablonka, P., Desai, V., et al. 2009, A\&A, 497, 713

Berrier, J. \& Sellwood, J. A. 2015, ApJ, 799, 213

Bett, P., Eke, V., Frenk, C. S., Jenkins, A., Helly, J. \& Navarro, J. 2007, MNRAS, 376, 215
Binney, J. \& Tremaine, S. 2008, Galactic Dynamics (2nd ed.; Princeton: Princeton University Press)

Bird, J. C., Kazantzidis, S., Weinberg, D. H., Guedes, J., Callegari, S., Mayer, L. \& Madau, P. 2013, ApJ, 773, 43

Block, D. L. \& Wainscoat, R. J. 1991, Nature, 353, 48

Blumenthal, G. R., Faber, S. M., Flores, R. \& Primack, J. R. 1986, ApJ, 301, 27

Bosma, A. 1996, in Barred Galaxies \& Circumnuclear Activity, eds. Sandqvist, Aa. \& Lindblad, P. O. (Heidelberg: Springer) p 67

Bournaud, F., Combes, F. \& Semelin, B. 2005, MNRAS, 364, L18

Bullock, J. S., Kolatt, T. S., Sigad, Y., et al. 2001, MNRAS, 321 , 559

Buta, R. \& Block, D. L. 2001, ApJ, 550, 243

Buta, R. J., Sheth, K., Athanassoula, E., et al. 2015, ApJS, 217, 32

Byrd, G. G., Valtonen, M. J., Valtaoja, L. \& Sundelius, B. 1986, A\&A, 166, 75

Cheung, E., Athanassoula, E., Masters, K. L., et al. 2013, ApJ, $\mathbf{7 7 9}, 162$ 
Christodoulou, D. M., Shlosman, I. \& Tohline, J. E. 1995, ApJ, 443, 551

Colín, P., Valenzuela, O. \& Klypin, A. 2006, ApJ, 644, 687

Courteau, S., Andersen, D. R., Bershady, M. A., MacArthur, L. A. \& Rix, H-W. 2003, ApJ, 594, 208

Danovich, M., Dekel, A., Hahn, O., Ceverino, D. \& Primack, J. 2015, MNRAS, 449, 2087

Davis, B. L., Berrier, J. C., Shields, D. W., et al. 2012, ApJS, 199, 33

Debattista, V. P. \& Sellwood, J. A. 2000, ApJ, 543, 704

Efstathiou, G. 1979, MNRAS, 187, 117

Efstathiou, G., Lake, G. \& Negroponte, J. 1982, MNRAS, 199, 1069

Elmegreen, D. M., Elmegreen, B. G. \& Bellin, A. D. 1990, ApJ, 364, 415

Erwin, P. 2005, MNRAS, 364, 283

Eskridge, P. B., Frogel, J. A., Pogge, R. W., et al. 2000, AJ, 119 536.

Gajda, G., Łokas, E. L. \& Athanassoula, E. 2016, 2016, arXiv:1606.00322

Gerin, M., Combes, F. \& Athanassoula, E. 1990, A\&A, 230, 37

Grogin, N. A., Kocevski, D. D., Faber, S. M., et al. 2011, ApJS, 197, 35

Guedes, J., Callegari, S., Madau, P. \& Mayer, L. 2011, ApJ, 742, 76

Hart, R. E., Bamford, S. P., Willett, K. W., et al. 2016, arXiv:1607.01019

Hernquist, L. 1990, ApJ, 356, 359

Koekemoer, A. M., Faber, S. M., Ferguson, H. C., et al. 2011, ApJS, 197, 36

Kormendy, J. 2013, In XXIII Canary Islands Winter School of Astrophysics, "Secular Evolution of Galaxies" eds. J.

Falcón-Barroso \& J. H. Knapen (Cambridge: Cambridge University Press), p. 1

Kormendy, J., Drory, N., Bender, R. \& Cornell, M. E. 2010, ApJ, 723, 54

Kormendy, J. \& Kennicutt, R. C. 2004, ARAA, 42, 603

Li, C., Gadotti, D. A., Mao, S. \& Kauffmann, G. 2009, MNRAS, 397, 726

Lin, Y., Cervantes Sodi, B., Li, C., Wang, L. \& Wang, E. 2014, ApJ, 796, 98

Lowing, B., Wang, W., Cooper, A., Kennedy, R., Helly, J., Cole, S. \& Frenk, C. 2015, MNRAS, 446, 2274

Lovelace, R. V. E. \& Hohlfeld, R. G. 1978, ApJ, 221, 51

Marinova, I. \& Jogee, S. 2007, ApJ, 659, 1176

Marinova, I., Jogee, S., Weinzirl, T., et al. 2012, ApJ, 746, 136

Martinez-Valpuesta, I., Shlosman, I. \& Heller, C. 2006, ApJ, 637 214

Masters, K. L., Nichol, R. C., Hoyle, B., et al. 2011, MNRAS, 411, 2026

Masters, K. L., Nichol, R. C., Haynes, M. P., et al. 2012, MNRAS, 424, 2180

Mathewson, D. S. \& Ford, V. L. 1996, ApJS, 109, 97

Matteucci, F. \& Francois, P. 1989, MNRAS, 239, 885
Mayer, L. \& Wadsley, J. 2004, MNRAS, 347, 277

Munshi, F., Governato, F., Brooks, A. M., et al. 2013, ApJ, 766, 56

Noguchi, M. 1987, MNRAS, 228, 635

Norman, C. A., Sellwood, J. A. \& Hasan, H. 1996, ApJ, 462, 114

Pfenniger, D. \& Norman, C. 1990, ApJ, 363, 391

Ostriker, J. P. \& Peebles, P. J. E. 1973, ApJ, 186, 467

Polyachenko, E., Berczik, P. \& Just, A. 2016, MNRAS, subiteed (arXiv:1601.06115)

Reese, A., Williams, T. B., Sellwood, J. A., Barnes, E. I. \&

Powell, B. A. 2007, AJ, 133, 2846

Romano-Díaz, E., Shlosman, I., Heller, C. \& Hoffman, Y. 2008, ApJL, 687, L13

Saha, K. \& Naab, T. 2013, MNRAS, 434, 1287

Salo, H. 1991, A\&A, 243, 118

Schaller, M., Frenk, C. S., Bower, R. G., et al. 2015, MNRAS, 451, 1247

Sellwood, J. A. 1989, MNRAS, 238, 115

Sellwood, J. A. 2003, ApJ, 587, 638

Sellwood, J. A. 2013, in Planets Stars and Stellar Systems, v.5, eds. T. Oswalt \& G. Gilmore (Heidelberg: Springer), 923 (arXiv:1006.4855)

Sellwood, J. A. 2014, arXiv:1406.6606 (on-line manual: http://www.physics.rutgers.edu/ sellwood/manual.pdf )

Sellwood, J. A. 2016, ApJ, 819, 92

Sellwood, J. A. \& Binney, J. J. 2002, MNRAS, 336, 785

Sellwood, J. A. \& Carlberg, R. G. 1984, ApJ, 282, 61

Sellwood, J. A. \& Debattista, V. P. 2006, ApJ, 639, 868

Sellwood, J. A. \& Evans, N. W. 2001, ApJ, 546, 176

Sellwood, J. A. \& McGaugh, S. S. 2005, ApJ, 634, 70

Sellwood, J. A. \& Moore, E. M. 1999, ApJ, 510, 125

Shen, J. \& Sellwood, J. A. 2006, MNRAS, 370, 2

Silk, J. \& Mamon, G. A. 2012, Res A\&GA, 12, 917

Skibba, R. A., Masters, K. L., Nichol, R. C., et al. 2012, MNRAS, 423, 1485

Snyder, G. F., Torrey, P., Lotz, J. M., et al. 2015, MNRAS, 454, 1886

Somerville, R. S. \& Davé, R. 2015, ARAA, 53, 51

Somerville, R. S., Popping, G. \& Trager, S. C. 2015, MNRAS, 453, 4337

Stinson, G. S., Brook, C., Macciò, A. V., Wadsley, J., Quinn, T.

R. \& Couchman, H. M. P. 2013, MNRAS, 428, 129

Toomre, A. 1963, ApJ, 138, 385

Toomre, A. 1969, ApJ, 158, 899

Toomre, A. 1981, in The Structure and Evolution of Normal Galaxies, ed. S. M. Fall \& D. Lynden-Bell (Cambridge: Cambridge University Press), p. 111

Wang, J., Kauffmann, G., Overzier, R., et al. 2011, MNRAS, 412, 1081

Weinberg, D. H., Bullock, J. S., Governato, F., Kuzio de Naray,

R. \& Peter, A. H. G. 2015, Proc. Nat. Acad. Sci., 112, 12249

Young, P. 1980, ApJ, 242, 1232

Zang, T. A. 1976, PhD thesis, MIT

\section{APPENDIX}

Sellwood \& McGaugh (2005) described how an isolated equilibrium spherical model could be compressed to include a second rigid mass component by assuming that the latter was added adiabatically. Their algorithm used the conservation of both radial action and angular momentum, as first set out by Young (1980), which therefore takes into account the extra resistance to compression caused by radial pressure that is ignored in algorithms that conserve angular momentum only (e.g. Blumenthal et al. 1986). Sellwood \& McGaugh (2005) also demonstrated that asphericity in the rigid mass had negligible effect, even when the rigid component was a disk, indicating that the change to the halo is very well determined only by the rigid mass enclosed within a spherical radius. We take the finite thickness of the disk into account when computing this mass, which spreads out the disk mass significantly only at radii that are within a couple of disk scale heights of the center. This algorithm yields the DF for the equilibrium halo in the composite disk+halo potential, from which halo particles can be selected. The final step of the set-up procedure is to realize the disk with particles.

Here we extend the algorithm to a three component system, in order to be able to create equilibrium bulges and halos in the presence of a disk. As for the single spherical component, the working assumption is that the DFs of the two spherical components, when expressed as functions of the actions, do not change as the combined model is assembled adiabatically. We therefore select models for the halo and bulge whose DFs when each is in isolation are 
known, or can be determined by Eddington inversion (Binney \& Tremaine 2008).

The first step is to construct 2D tables for the specific energy, $E$, as functions of the two actions: specific angular momentum, $L$, and radial action, $J_{r}$, in the potential well of each isolated spherical component. These tables are needed so that the DF, which is usually expressed as a function of the classical integrals, $f(E, L)$, can be determined from known values of the actions.

The solution for the equilibrium of the composite system proceeds iteratively. Using the total spherical potential of the composite system at each iteration, we compute an additional 2D table of $J_{r}\left(E^{\prime}, L\right)$, where $E^{\prime}$ is a possible energy. To evaluate the DF for either component for some given values of $\left(E^{\prime}, L\right)$, we use our tables first to map $\left(E^{\prime}, L\right) \rightarrow\left(J_{r}, L\right)$ and then $\left(J_{r}, L\right) \rightarrow(E, L)$, and set $f\left(E^{\prime}, L\right)=f(E, L)$. We can therefore integrate the DF of both spherical components over all accessible values of $E^{\prime}$ and $L$ to construct revised estimates of their densities. The new density estimates yield an improved estimate of the combined potential, with the inclusion of the disk monopole term, from which an improved table of $J_{r}\left(E^{\prime}, L\right)$ may be computed. The procedure generally converges to an acceptable level in 10-15 iterations.

This procedure can be generalized to determine equilibrium DFs for separate components in any composite system, with or without rigid potentials. 